Article

\title{
Convergent Double Auction Mechanism for a Prosumers' Decentralized Smart Grid
}

\author{
Tadahiro Taniguchi ${ }^{1, *}$, Tomohiro Takata ${ }^{2}$, Yoshiro Fukui ${ }^{1}$ and Koki Kawasaki ${ }^{3}$ \\ Received: 2 August 2015 ; Accepted: 19 October 2015 ; Published: 30 October 2015 \\ Academic Editor: G.J.M. (Gerard) Smit \\ ${ }^{1}$ College of Information Science and Engineering, Ritsumeikan University, 1-1-1 Noji Higashi, Kusatsu, \\ Shiga 525-8577, Japan; fukui@em.ci.ritsumei.ac.jp \\ 2 Research Organization of Science and Technology, Ritsumeikan University, 1-1-1 Noji Higashi, Kusatsu, \\ Shiga 525-8577, Japan; takata@em.ci.ritsumei.ac.jp \\ ${ }^{3}$ Graduate School of Information Science and Engineering, Ritsumeikan University, 1-1-1 Noji Higashi, \\ Kusatsu, Shiga 525-8577, Japan; kawasaki@em.ci.ritsumei.ac.jp \\ * Correspondence: taniguchi@em.ci.ritsumei.ac.jp; Tel.: +81-77-561-5839; Fax: +81-77-561-5745
}

\begin{abstract}
In this paper, we propose a novel automated double auction mechanism called convergent linear function submission-based double-auction (CLFS-DA) for a prosumers' decentralized smart grid. The target decentralized smart grid is a regional electricity network that consists of many prosumers that have a battery and a renewable energy-based generator, such as photovoltaic cells. In the proposed double-auction mechanism, each intelligent software agent representing each prosumer submits linear demand and supply functions to an automated regional electricity market where they are registered. It is proven that the CLFS-DA mechanism is guaranteed to obtain one of the global optimal price profiles in addition to it achieving an exact balance between demand and supply, even through the learning period. The proof of convergence is provided on the basis of the theory of LFS-DA, which gives a clear bridge between a function submission-based double auction and a dual decomposition (DD)-based real-time pricing procedure. The performance of the proposed mechanism is demonstrated numerically through a simulation experiment.
\end{abstract}

Keywords: distributed algorithm; double auction; dual decomposition (DD); Lagrangian relaxation; multi-agent system

\section{Introduction}

\subsection{Prosumers' Decentralized Smart Grid}

Electricity network concepts employing emergent smart grid technologies are gathering attention [1-6]. Reducing our dependency on thermal power generation based on fossil fuel and introducing renewables-based distributed energy resources (DERs), such as photovoltaic (PV) cells, wind turbines and batteries, into our residential areas will change our total electric power systems in the future [7]. Various operation, optimization and management methods for distributed energy systems have been studied in the context of smart grids [8-12]. Originally, the domain of smart grids was an interdisciplinary research field involving power electronics, information science, control theory and economics $[3,13,14]$. In electricity networks, balancing demand and supply is crucial. To balance demand and supply in residential electricity networks based on renewables-based DERs, the information technologies and economics methods that encourage suppliers and consumers to behave so as to stabilize the network and to maximize its social welfare are promising. In this context, market-based decentralized control methods are attracting attention in the research field of smart grids [1-5,15-18]. 
In this paper, we focus on the market-based approach to demand-side management (DSM) for prosumers' electricity networks. The term prosumer is defined as an entity that is a producer and a consumer simultaneously [19-23]. We investigate a regional prosumers' decentralized smart grid, called an inter-intelligent renewable energy network (i-Rene) [21,24]. The overview of i-Rene is shown in Figure 1. Each house in i-Rene has a renewable energy-based generator and battery. A smart meter containing a software agent can trade electricity automatically through a regional electricity market. The smart meter also manages battery and shiftable devices [25-27] using smart grid technologies. The electricity network itself is connected to other outside electricity networks through a gateway. The prosumers have the option to buy electricity from an outside grid shown in Figure 1 . The size of i-Rene is assumed to be between about ten houses and several thousand. Developing a DSM method that can balance demand and supply in a prosumers' electricity network is thus one of the central topics in this research field.
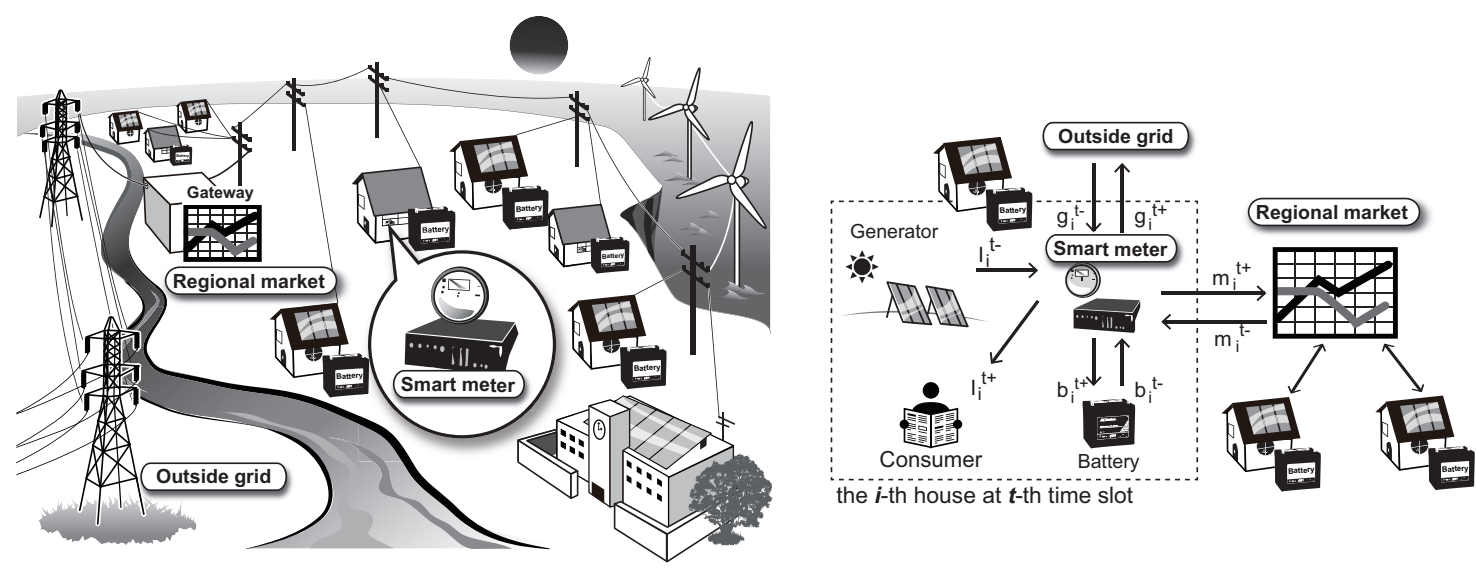

Figure 1. Overview of the inter-intelligent renewable energy network (i-Rene) (left) and the power flow and variable definitions in the i-Rene mathematical model (right) [21].

\subsection{Market-Based Demand-Side Management (DSM)}

Numerous studies of market-based DSM have been conducted for conventional electricity networks, i.e., generators and consumers exist separately in an electricity grid. Game theory and control theory have also given the theoretical foundation to market-based DSM [4,13,16,28-34]. Moreover, a comprehensive survey of studies of DSM was provided by Alizadeh et al. [3].

Saad et al. [13] conducted a survey of game theory-based approaches to electricity networks. Various mechanisms and their game-theoretic properties, such as the Nash equilibrium and incentive compatibility, have been provided [16,28,29],

From the viewpoint of control theory, dual decomposition (DD) has been used as a key theoretical component that connects social welfare maximization problems to real-time pricing (RTP). Palomar provided a comprehensive tutorial of DD [35]. DD relaxes a network constraint in a primal problem, such as a social welfare maximization problem, based on the method of the Lagrange multiplier. In this approach, Lagrange multipliers are found to be "price profiles". Based on the DD framework, many RTP mechanisms have been studied [4,30-34]. Most of the RTP mechanisms are guaranteed to bring the system to one of the optimal solutions, i.e., each of them usually has proof of convergence (see the Appendix).

However, most of the control theory-based RTP mechanisms have undesirable assumptions. First, they assume that a network has a central price controller, e.g., a utility company, who dedicatedly tries to maximize social welfare. It is not assumed to behave selfishly. The assumption of an irrational, i.e., unselfish, player can become the vulnerability of a real social and economic system. Ideally, it should be avoided. Second, the mechanisms relax the network constraint, i.e., 
the balance of demand and supply. Therefore, the balance of demand and supply is not satisfied until the iterative optimization process between consumers and the utility converges after an infinite number of iterative communications between them, although the balance of demand and supply is essentially important in electricity networks.

On the other hand, double auction-based mechanisms usually satisfy the constraint about the balance of demand and supply, because the electricity price is determined to balance demand and supply and not to maximize social welfare. PowerMatcher by Kok et al. $[1,36]$ is one of the most important works and field tests that exploit double auction for distributing electricity. PowerMatcher is a smart grid technology that consists of a distributed multi-agent software system that conducts market-based integration of relatively small-sized DER units. They used a function submission-based double auction (FS-DA) for the simplicity of the implementation. In an FS-DA, each agent submits demand and supply functions to a market. The auctioneer that receives the functions outputs a clearing price by determining the intersection of aggregate demand and supply functions. This procedure does not require iterative communication between the agents and the auctioneer.

Recently, Taniguchi et al. [21] described the theoretical relation between a linear FS-DA (LFS-DA) and a DD. The LFS-DA is an FS-DA in which only linear demand and supply functions are used. They proved that price updates in the LFS-DA become mostly equal to that of the RTP based on a DD with a sub-gradient method (RTP-DDSG), basically, except for a constant factor. This constructed a clear bridge between the double auction-based approach and the DD-based approach. The LFS-DA was shown to increase the social welfare of an electricity network in a similar way as the RTP-DDSG. However, the LFS-DA, generally, cannot bring the system to one of the optimal solutions, i.e., the LFS-DA does not have proof of convergence. If all of the prosumers behave arbitrarily, the price profile might become even worse than the initial state. In many cases, the LFS-DA outperforms conventional RTP-DDSG in i-Rene. However, for stable operation of a prosumers' decentralized smart grid, a double auction mechanism that has a convergence proof is definitely desirable.

\subsection{Convergent Double Auction Mechanism}

Based on this background, in this paper, we propose a new double auction mechanism for i-Rene called the convergent LFS-DA (CLFS-DA), which is an extension of the LFS-DA market mechanism. In contrast to the LFS-DA, which does not have convergence proof, the CLFS-DA guarantees that the price profile of electricity converges to an optimal price profile and maximizes social welfare without any central controller, e.g., a utility or an administrative organization. The maximization of social welfare was surely performed entirely in a bottom-up manner.

The essential characteristics of three comparative methods are listed in Table 1. "Fully decentralized" means that the mechanism does not require a central player that is expected to behave unselfishly, i.e., dedicatedly and tries to maximize social welfare. "Balance of demand and supply" means that balance of demand and supply is satisfied throughout its learning phase, i.e., iterative communication between a central market and agents. "Convergence" means that the method has the proof of convergence and is expected to maximize social welfare. Among the three comparative methods, only the CLFS-DA has all of the characteristics.

Table 1. Characteristics of the related mechanisms. RTP-DDSG: real-time pricing dual decomposition with a sub-gradient method; LFS-DA: linear function submission based double-auction; CLFS-DA: convergent linear function submission-based double-auction.

\begin{tabular}{cccc}
\hline Characteristics & RTP-DDSG & LFS-DA & CLFS-DA \\
\hline Fully decentralized & - & $\checkmark$ & $\checkmark$ \\
Balance of demand and supply & - & $\checkmark$ & $\checkmark$ \\
Convergence & $\checkmark$ & - & $\checkmark$ \\
\hline
\end{tabular}


The difference between the LFS-DA and the CLFS-DA introduced in Section 3 might look simple and small in its formulation at a glance. However, the difference between the LFS-DA and the CLFS-DA is qualitative and crucial. The extension is not trivial from the viewpoint of the double auction mechanism. It gives a significant and qualitative difference to the performance of the mechanism, i.e., the convergence property is satisfied. Convergence proof is crucial when we decide to adopt a market-based DSM method for designing a prosumers' decentralized smart grid. A mechanism without convergence proof might use electricity inefficiently and decrease social welfare in the network. We also show that the CLFS-DA outperforms the LFS-DA from a quantitative viewpoint.

Our main and novel contribution is as follows:

We propose a CLFS-DA mechanism for a regional prosumers' decentralized smart grid. The mechanism has convergence proof in addition to all of the advantageous properties of the LFS-DA, i.e., an exact balance between demand and supply and completely decentralized operation.

This means that the CLFS-DA is superior to the LFS-DA in all aspects, although it has been shown that the LFS-DA outperforms the RTP-DDSG in i-Rene [21]. Several related works treat the FS-DA $[17,37,38]$. One of the most closely-related works was conducted by Papavasiliou et al. [38], who proposed a Newton algorithm-based double auction mechanism. The mechanism by Papavasiliou et al. is interpreted as a Newton algorithm with a decomposable structure. However, they did not treat prosumers' electricity networks where each prosumer uses renewable energy resources and batteries and is allowed to become a consumer and a supplier.

The remainder of this paper is organized as follows. Section 2 provides the problem definitions and model description of i-Rene, the target regional electricity network. The primal problem is defined as a maximization problem of social welfare, i.e., a Benthamite social welfare function, and a conventional RTP-DDSG mechanism is introduced. In Section 3, the CLFS-DA mechanism is proposed, and its convergence proof is provided. In addition, the characteristics of the mechanism are described. Section 4 presents the details of a simulation experiment and its results. Section 5 concludes this paper.

\section{Model and Conventional Real-Time Pricing (RTP)}

\subsection{Basic Assumptions of $i$-Rene}

A target decentralized smart grid, i-Rene, is defined as follows (Figure 1). Electricity suppliers and consumers are not distinguished. Each agent has a generator and a storage device. Each agent also has a smart meter that runs a software system that controls the electricity of the house automatically. It manages its battery, transmits electricity to other agents, and communicates with other information systems. Hereafter, the prosumers living in the house and the software system are simply referred to as an agent.

In the regional decentralized smart grid, buildings are connected via a regional electricity market. Through the market, each prosumer can buy and sell electricity at a variable price. The local electricity price fluctuates depending on demand and supply. An optional alternative is provided by the outside grid. At any moment, agents can buy electricity at the fixed price from the outside grid. Furthermore, surplus electricity can be sold to the outside grid. However, the price is fixed and low. It is assumed that the outside grid is a conventional unilateral grid, which is common especially in Japan.

Suppose that i-Rene has $N$ agents. A set of the agents is defined as $\mathcal{N}:=\{1,2, \ldots, N\}$. Each agent is able to charge, discharge, consume, generate, sell and buy electricity through its smart meter at every time slot. The number of time slots is common among all of the agents. The set of time slots is represented by $\mathcal{T}:=\{1,2, \ldots, T\}$. The $i$-th agent consumes $l_{i}^{t+}$, generates $l_{i}^{t-}$, charges $b_{i}^{t+}$, discharges $b_{i}^{t-}$, sells to $m_{i}^{t+}$, buys from the regional market $m_{i}^{t-}$, sells to $g_{i}^{t+}$ and buys from the outside grid $g_{i}^{t-}$ during each time slot $t$. Based on the definitions, the $i$-th agent's state vector for the time slot $t$ is 
defined as $x_{i}^{t}:=\left(l_{i}^{t+}, l_{i}^{t-}, b_{i}^{t+}, b_{i}^{t-}, m_{i}^{t+}, m_{i}^{t-}, g_{i}^{t+}, g_{i}^{t-}\right)$. Each variable has its upper limit and/or lower limit on the basis of the limited capability of each device or systems (see the Nomenclature). The superscripts . ${ }^{-}$and ${ }^{+}$represent the direction of power flows from a smart meter, which is located at the center of energy flows in a house. Basically, $\cdot^{-}$and $\cdot^{+}$represent inflow and outflow, respectively. It is assumed that the law of the conservation of energy is satisfied for each time slot $t$ about the power flows through the smart meter as follows:

$$
h^{t}\left(x_{i}^{t}\right):=l_{i}^{t+}-l_{i}^{t-}+b_{i}^{t+}-b_{i}^{t-}+m_{i}^{t+}-m_{i}^{t-}+g_{i}^{t+}-g_{i}^{t-}=0
$$

The amount of electricity demanded has to be balanced against the supply in the decentralized smart grid for each time slot $t$ :

$$
f_{i}^{t}\left(x_{i}\right):=\gamma m_{i}^{t+}-m_{i}^{t-}, \quad \sum_{i \in \mathcal{N}} f_{i}^{t}\left(x_{i}\right)=0
$$

where the electricity transmission efficiency is represented by $\gamma \in[0,1]$.

The storage profile $s_{i}^{t}$ represents the state of charge (SOC) of the $i$-th agent's storage device at time $t$. The dynamics of the storage device is given as follows:

$$
s_{i}^{t}:=s_{i}^{t-1}+\eta_{i} b_{i}^{t+}-b_{i}^{t-}=s_{i}^{\text {init }}+\sum_{k \in\{1,2, \ldots, t\}}\left(\eta_{i} b_{i}^{k+}-b_{i}^{k-}\right)
$$

where the initial SOC of the $i$-th agent's storage device is represented by $s_{i}^{\text {init }}$, and the storage efficiency is represented by $\eta_{i} \in[0,1]$. If $\eta_{i}=1$, the $i$-th agent can fully charge electricity without any loss. As a whole, the $i$-th agent's feasible set $\mathcal{X}_{i}$, except for the network constraint Equation (2), is described as follows:

$$
\begin{gathered}
\mathcal{X}_{i}:=\left\{x_{i} \in \mathbb{R}^{8 T} \mid\right. \text { Constraints described in the Nomenclature } \\
\left.\qquad h^{t}\left(x_{i}^{t}\right)=0 \quad \forall t \in \mathcal{T}\right\}
\end{gathered}
$$

where $x_{i}:=\left(x_{i}^{t}\right)_{t \in \mathcal{T}}$.

Agents can sell electricity to the outside grid at the price of $p_{t}^{G+}$, and buy electricity from it at the price of $p_{t}^{G-}$ per unit, respectively. If reverse power flow is completely prohibited, $p_{t}^{G+}$ should be zero. To avoid obvious and meaningless solution, i.e., immediate resale behavior, we athe following assumption:

$$
0 \leq p_{t}^{G+} \leq p_{t}^{G-}
$$

\subsection{Primal Problem}

The cost for electricity generation $l_{i}^{t-}$ for the $i$-th agent is defined as $C_{i}^{t}: \mathbb{R} \rightarrow \mathbb{R}$, where $C_{i}^{t}$ is a convex function of class $C^{2}$. The utility for consuming electric energy $l_{i}^{t+}$ for the $i$-th agent is represented by $D_{i}^{t}: \mathbb{R} \rightarrow \mathbb{R}$, where $D_{i}^{t}$ is a concave function of class $C^{2}$. The individual welfare $W_{i}: \mathbb{R}^{8 T} \times \mathbb{R}^{T} \rightarrow \mathbb{R}$ of the $i$-th agent is defined as follows:

$$
\begin{aligned}
W_{i}\left(x_{i}, p\right) & :=\sum_{t \in \mathcal{T}} W_{i}^{t}\left(x_{i}^{t}, p_{t}\right) \\
W_{i}^{t}\left(x_{i}^{t}, p_{t}\right) & :=\phi_{i}^{t}\left(x_{i}^{t}\right)+p_{t} \gamma m_{i}^{t+}-p_{t} m_{i}^{t-} \\
\phi_{i}^{t}\left(x_{i}^{t}\right) & :=D_{i}^{t}\left(l_{i}^{t+}\right)-C_{i}^{t}\left(l_{i}^{t-}\right)+p_{t}^{G+} g_{i}^{t+}-p_{t}^{G-} g_{i}^{t-}
\end{aligned}
$$

where $x_{i}:=\left(x_{i}^{t}\right)_{t \in \mathcal{T}}, p:=\left(p_{t}\right)_{t \in \mathcal{T}}=\left(p_{1}, \ldots, p_{T}\right), p_{t}$ is a buyer's price in the market at time $t \in \mathcal{T}$ and $\phi_{i}^{t}\left(x_{t}\right)$ is a utility function of the $i$-th agent at time $t$ that includes payments to the outside grid. The seller's price and the buyer's price are usually different, because there is electricity energy loss during transmission. To balance the amount of money paid by buyers and sellers, the seller's price 
can be determined to be $\gamma p_{t}$. The social welfare of the prosumers' decentralized smart grid is defined by following $W(x, p)$ :

$$
\begin{aligned}
W(x, p) & :=\sum_{i \in \mathcal{N}} W_{i}\left(x_{i}, p\right) \\
& =\sum_{t \in \mathcal{T}}(\sum_{i \in \mathcal{N}} \phi_{i}^{t}\left(x_{i}\right)+p_{t} \underbrace{\sum_{i \in \mathcal{N}}\left(\gamma m_{i}^{t+}-m_{i}^{t-}\right)}_{=0 \text { from Equation (2) }}) \\
& =\sum_{t \in \mathcal{T}}\left(\sum_{i \in \mathcal{N}} \phi_{i}^{t}\left(x_{i}\right)\right)
\end{aligned}
$$

where $x:=\left(x_{i}\right)_{i \in \mathcal{N}}=\left(x_{1}, \ldots, x_{N}\right)$. This means that the social welfare of $\mathrm{i}$-Rene is not affected by the price profile of the market. Maximizing $W(x, p)$ is the same problem as maximizing $\phi(x):=$ $\sum_{i \in \mathcal{N}} \phi_{i}\left(x_{i}\right)$ where $\phi_{i}\left(x_{i}\right):=\sum_{t \in \mathcal{T}} \phi_{i}^{t}\left(x_{i}^{t}\right)$.

The following problem is defined as the primal problem, which aims to maximize the social welfare of the regional network.

Problem 1 (Primal problem).

$$
\begin{aligned}
\underset{x \in \mathbb{R}^{8 N T}}{\operatorname{maximize}} & \phi(x)=\sum_{i \in \mathcal{N}} \phi_{i}\left(x_{i}\right) \\
\text { subject to } & x_{i} \in \mathcal{X}_{i} \quad \forall i \in \mathcal{N} \\
& \sum_{i \in \mathcal{N}} f_{i}^{t}\left(x_{i}\right)=0 \quad \forall t \in \mathcal{T}
\end{aligned}
$$

However, any entity cannot obtain information about $\left\{C_{i}^{t}, D_{i}^{t}\right\}_{i \in \mathcal{N}, t \in \mathcal{T}}$, because they are usually private information. In addition, any entity cannot control the behavior of the agents directly, because they are independent rational players. If the central control system could perform such things, to solve the primal problem shown above requires an exponentially large computational cost when $N$ becomes large. That is, centrally solving the primal problem is realistically impossible. Based on this background, a decentralized optimization method is required. A decentralized optimization method using price information is derived via a dual problem.

\subsection{Dual Decomposition (DD)}

\subsubsection{Dual Problem}

Network optimization problems like the target primal problem can be decomposed via a dual problem. Such a procedure is called dual decomposition (DD) [35]. DD transforms a primal problem into many independent sub-problems that can be solved by each agent's rational behavior and a master problem that is solved by optimizing price profiles. The solutions of the primal problem and the dual problem become the same if the conditions satisfy Slater's theorem [39]. The primal problem satisfies this theorem. The DD provides the theoretical basis for RTP. The target primal problem in Equation (12) can be transformed into a dual problem as follows.

Problem 2 (Dual problem).

$$
\begin{aligned}
& \underset{p \in \mathbb{R}^{T}}{\operatorname{minimize}} g(p) \\
& g(p):=\sum_{i \in \mathcal{N}} \sup _{i} \in \mathcal{X}_{i} L_{i p}\left(x_{i}\right) \\
& L_{i p}\left(x_{i}\right):=\phi_{i}\left(x_{i}\right)+\sum_{t \in \mathcal{T}} p_{t} f_{i}^{t}\left(x_{i}\right)
\end{aligned}
$$


The Lagrange multiplier $p:=\left(p_{t}\right)_{t \in \mathcal{T}}$ means the "price" of goods that are constrained by an equation $f_{i}^{t}$ which is relaxed by $p_{t}$ [35]. In this problem, $p_{t}$ becomes the price of electricity in the market at time $t$.

\subsubsection{Sub-Problems}

The objective function Equation (16) of the dual problem is divided into $N$ objective functions if the Lagrange multipliers $p$ are introduced. Each agent can solve the maximization problem of each objective function independently.

Problem 3 (Sub-problems).

$$
\begin{aligned}
& \underset{x_{i} \in \mathbb{R}^{8 T}}{\operatorname{maximize}} L_{i p}\left(x_{i}\right) \\
& \text { subject to } x_{i} \in \mathcal{X}_{i}
\end{aligned}
$$

Each agent is expected to solve each sub-problem autonomously if we can regard each agent as a rational agent. One of the optimal solutions $x_{i}^{*}(p)$ to the problem Equation (18) is described as follows:

$$
x_{i}^{*}(p)=\underset{x_{i} \in \mathcal{X}_{i}}{\operatorname{argmax}} L_{i p}\left(x_{i}\right)
$$

where $x_{i}^{* t}=\left(l_{i}^{* t+}, l_{i}^{* t-}, b_{i}^{* t+}, b_{i}^{* t-}, m_{i}^{* t+}, m_{i}^{* t-}, g_{i}^{* t+}, g_{i}^{* t-}\right)$ and $x_{i}^{*}=\left(x_{i}^{* t}\right)_{t \in \mathcal{T}}$.

\subsubsection{Master Problem}

We assume there is a central entity that determines the price profile in the market. Based on the agents' optimal strategies $\left(x_{i}^{*}(p)\right)_{i \in \mathcal{N}}$, we assume that the central entity tries to solve the following problem.

Problem 4 (Master problem).

$$
\begin{aligned}
& \underset{p \in \mathbb{R}^{T}}{\operatorname{minimize}} g(p) \\
& \quad g(p)=\sum_{i \in \mathcal{N}} L_{i p}\left(x_{i}^{*}(p)\right)
\end{aligned}
$$

This problem is called the master problem. Solving this problem corresponds to searching for an adequate price profile that could balance demand and supply.

If we determine the solution of a master problem and sub-problems simultaneously, the solution becomes that of the dual problem. Iterative optimization techniques can achieve such an optimization in practice.

\subsubsection{Real-Time Pricing based on a Dual Decomposition with a Sub-Gradient Method (RTP-DDSG)}

Based on the DD framework, a conventional RTP mechanism, i.e., RTP-DDSG, can be derived. In the RTP-DDSG, each agent tries to maximize its welfare rationally by solving its sub-problem, and the central entity updates the price profile [4]. The central controller updates the price profile $p_{t}^{(k)}$ using the sub-gradient descent $[4,35]$.

$$
\begin{aligned}
p_{t}^{(k+1)} & =p_{t}^{(k)}-\theta_{k}^{t} \xi_{t}\left(p^{(k)}\right) \\
\xi_{t}\left(p^{(k)}\right) & :=\sum_{i \in \mathcal{N}} f_{i}^{t}\left(x_{i}^{*}\left(p^{(k)}\right)\right) \\
& =\sum_{i \in \mathcal{N}}\left(\gamma m_{i}^{* t+}\left(p^{(k)}\right)-m_{i}^{* t-}\left(p^{(k)}\right)\right)
\end{aligned}
$$


where $\theta_{k}^{t}>0$ is the learning rate. By updating of $x_{i}$ and $p$ iteratively, the numerical solution of the dual problem in Equations (15)-(17) can be obtained. This numerical solution yields a solution of the original primal problem in Equations (12)-(14). Therefore, the RTP-DDSG is expected to maximize social welfare of the network under some conditions. Convergence proof of the RTP-DDSG is provided in the Appendix.

\section{Convergent Linear Function Submission-Based Double-Auction (CLFS-DA)}

\subsection{Overview}

Algorithm 1 shows the overall market mechanism of the CLFS-DA, which is our proposal in this paper, in the form of pseudocode. The CLFS-DA is an extension of the LFS-DA proposed in [21]. In the LFS-DA, its iterative process is not guaranteed to converge to the optimal value and solution because the LFS-DA is a fully-decentralized mechanism, and the demand and supply function submitted to a regional market is arbitrarily controlled by each agent independently. In the LFS-DA, any rules for $\beta_{i}^{t}$ are not settled [21]. The parameter $\beta_{i}^{t}$ is fixed only for its analytical purpose in [21]. Based on the backgrounds, we extend the LFS-DA and obtain the CLFS-DA, which contains a rule for determining $\beta_{i}^{t}$ for each agent. Surprisingly, the very simple modification of the mechanism enables us to provide the proof of convergence. Most of the algorithm of the CLFS-DA, except for a rule controlling $\beta_{i}^{t}$, is the same as that of the LFS-DA.

\subsection{Transactions with the Convergent Linear Function Submission-Based Double-Auction (CLFS-DA)}

In the CLFS-DA, each agent $i$ has a supply function $m_{i}^{t+}=\mu_{i}^{t+}\left(p_{t}\right)$ and a demand function $m_{i}^{t-}=\mu_{i}^{t-}\left(p_{t}\right)$ for each time slot $t$. We assume that the two functions are determined on the basis of the two parameters $\alpha_{i}^{t}$ and $\beta_{i}^{t}$ :

$$
\begin{aligned}
& m_{i}^{t+}=\mu_{i}^{t+}\left(p_{t}\right):=\left\lfloor\beta_{i}^{t} p_{t}-\alpha_{i}^{t}\right\rfloor \\
& m_{i}^{t-}=\mu_{i}^{t-}\left(p_{t}\right):=\left\lfloor-\beta_{i}^{t} p_{t}+\alpha_{i}^{t}\right\rfloor
\end{aligned}
$$

where $\lfloor x\rfloor:=\max (x, 0)$. The functions, i.e., Equations (25) and (26), are essentially generated from a single linear function.

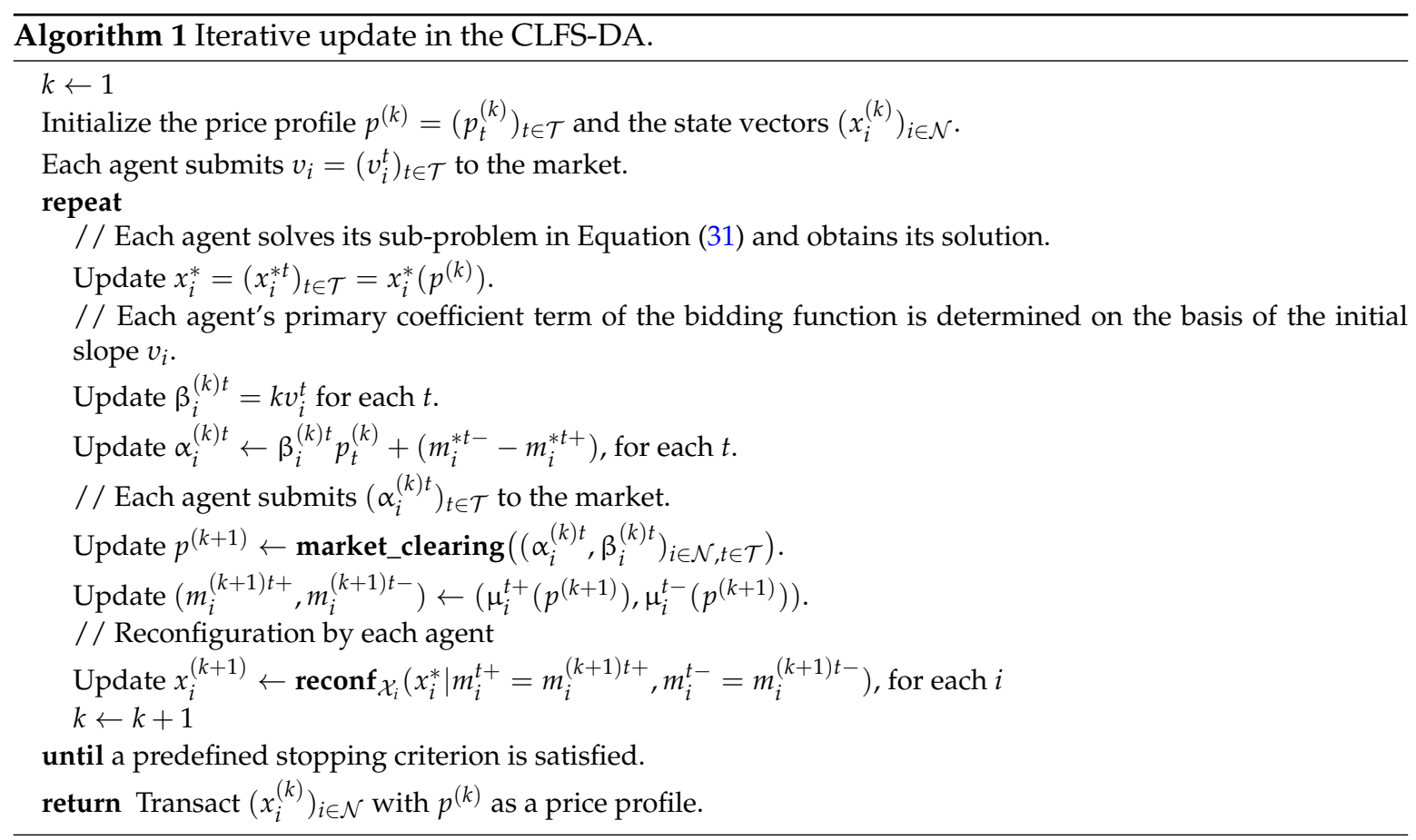


In the CLFS-DA, the $i$-th agent submits the initial slope of the bidding function $\left(v_{i}^{t}\right)_{t \in \mathcal{T}}$ to the regional market when a day-ahead market starts. Once the iterative procedure of the day-ahead market starts, $\beta_{i}^{t}$ is determined based on the initial slope parameter, i.e., $\beta_{i}^{t}=k v_{i}^{t}$ throughout the iterative process of the day-ahead market in the CLFS-DA. This is the main difference between the LFS-DA and the CLFS-DA. Therefore, $\beta_{i}^{t}$ itself does not converge to a certain value, but diverges to infinity by definition. From the viewpoint of an ordinary double auction mechanism, the demand and supply functions submitted to a market are assumed to have information about the agent's marginal utility function. Therefore, many people, who are familiar with basic microeconomics to some extent, expect that $\beta_{i}^{t}$ should converge to a certain value through an iterative process. In contrast, the proposed mechanism, the CLFS-DA, requires $\beta_{i}^{t}$ to diverge to infinity. In this way, CLFS-DA is a non-trivial mechanism from the viewpoint of microeconomics and double auction.

Each agent does not need to submit $\beta_{i}^{t}$ to the auctioneer in each iteration. In each iteration, each agent submits $\alpha_{i}^{t}$ to the auctioneer of the decentralized smart grid in order to provide the demand and supply functions of each agent for each time slot $t \in \mathcal{T}$. The parameter $\alpha_{i}^{t}$ is a flexible variable, and each agent updates it at each iteration to optimize his/her individual welfare function.

After the auctioneer receives the demand and supply functions of all participants of the regional electricity market in the $k$-th iteration, price profile $p$ is determined by finding the point where the aggregate supply and demand functions intersect to clear the market by balancing supply and demand completely. The constraint for balancing supply and demand in Equation (2) becomes:

$$
\begin{aligned}
\sum_{i \in \mathcal{N}} f_{i}^{t}\left(x_{i}, p_{t}\right) & =\gamma \sum_{i \in \mathcal{N}}\left\lfloor\beta_{i}^{t} p_{t}-\alpha_{i}^{t}\right\rfloor-\sum_{i \in \mathcal{N}}\left\lfloor-\beta_{i}^{t} p_{t}+\alpha_{i}^{t}\right\rfloor \\
& =\gamma \sum_{i \in I_{t}^{+}\left(p_{t}\right)}\left(\beta_{i}^{t} p_{t}-\alpha_{i}^{t}\right)-\sum_{i \in I_{t}^{-}\left(p_{t}\right)}\left(-\beta_{i}^{t} p_{t}+\alpha_{i}^{t}\right)=0
\end{aligned}
$$

where we denote the set of consumers at time $t$ by $I_{t}^{-}\left(p_{t}\right):=\left\{i \in \mathcal{N} \mid \frac{\alpha_{i}^{t}}{\beta_{i}^{t}}>p_{t}\right\}$ and the set of suppliers at time $t$ by $I_{t}^{+}\left(p_{t}\right):=\left\{i \in \mathcal{N} \mid \frac{\alpha_{i}^{t}}{\beta_{i}^{t}} \leq p_{t}\right\}$. In this case, based on the constraint in Equation (27), a clearing price satisfies the following equation:

$$
p_{t}=\left(\gamma \alpha_{I_{t}^{+}\left(p_{t}\right)}^{t}+\alpha_{I_{t}^{-}\left(p_{t}\right)}^{t}\right) /\left(\gamma \beta_{I_{t}^{+}\left(p_{t}\right)}^{t}+\beta_{I_{t}^{-}\left(p_{t}\right)}^{t}\right)
$$

where $\alpha_{I_{t}^{+}\left(p_{t}\right)}^{t}:=\sum_{i \in I_{t}^{+}\left(p_{t}\right)} \alpha_{i^{\prime}}^{t} \quad \alpha_{I_{t}^{-}\left(p_{t}\right)}^{t}:=\sum_{i \in I_{t}^{-}\left(p_{t}\right)} \alpha_{i}^{t}, \beta_{I_{t}^{+}\left(p_{t}\right)}^{t}:=\sum_{i \in I_{t}^{+}\left(p_{t}\right)} \beta_{i^{\prime}}^{t}$ and $\beta_{I_{t}^{-}\left(p_{t}\right)}^{t}:=\sum_{i \in I_{t}^{-}\left(p_{t}\right)} \beta_{i}^{t}$. The price $p_{t}$ satisfying Equation (29) balances supply and demand exactly and fulfills the constraint in Equation (27). It is known that the clearing price $p_{t}$ that satisfies Equation (29) uniquely exists. It can be calculated exactly [21].

In the CLFS-DA, when $\left(\alpha_{i}^{t}\right)_{i \in \mathcal{N}, t \in \mathcal{T}}$ from all of the agents are received by the auctioneer at the $k$-th iteration, the price $p_{t}^{(k+1)}$ is determined by solving Equation (29). Each agent is requested either to transmit $m_{i}^{(k+1) t+}=\mu_{i}^{t+}\left(p_{t}^{(k+1)}\right)$ or to receive $m_{i}^{(k+1) t-}=\mu_{i}^{t-}\left(p_{t}^{(k+1)}\right)$ on the basis of the price $p_{t}^{(k+1)}$. Each agent has to reconfigure its $x_{i}^{t}$ for the given $\left(m_{i}^{(k+1) t+}, m_{i}^{(k+1) t-}\right)$ so as to satisfy the constraint $x_{i} \in \mathcal{X}_{i}$ except for the inequality constraints about $m_{i}^{t+}$ and $m_{i}^{t-}$ after $\left(m_{i}^{(k+1) t+}, m_{i}^{(k+1) t-}\right)$ is determined. We assume that the system allows the agents to use the margin of network capacity when $\left(m_{i}^{(k+1) t+}, m_{i}^{(k+1) t-}\right)$, occasionally, violate their inequality constraints. After the iterative mechanism satisfies a predefined stopping criterion, the participants transact real electricity and money. Under the mechanism, each agent is expected to behave selfishly, i.e., rationally. 


\subsection{Iterative Process of the Convergent Linear Function Submission-Based Double-Auction (CLFS-DA)}

Through the communication made iteratively between an auctioneer and participants, the price profile $p$ and state vectors $\left\{x_{i}^{(k)}\right\}_{i \in \mathcal{N}}$ gradually change. During the iteration phase in a day-ahead market, $\beta_{i}^{t}$ and $p_{t}$ are given by the auctioneer. Note that in the $k$-th iteration, $\beta_{i}^{(k) t}=k v_{i}^{t}$ and $v_{i}^{t}$ cannot be changed once the $i$-th agent submits it to the auctioneer in the initial step of the mechanism. Based on the externally-determined parameters, $\alpha_{i}^{t}$ is determined uniquely from $\left(m_{i}^{t+}, m_{i}^{t-}\right)$ as follows:

$$
\alpha_{i}^{t}=\beta_{i}^{t} p_{t}+\left(m_{i}^{t-}-m_{i}^{t+}\right)
$$

Based on Equations (25) and (26), $\left(m_{i}^{t+}, m_{i}^{t-}\right)$ is also determined uniquely from $\alpha_{i}^{t}$ under the condition that $\beta_{i}^{t}$ and $p_{t}$ are fixed. Therefore, optimizing $\left(\alpha_{i}^{t}\right)_{t \in \mathcal{T}}$ and optimizing $\left(m_{t}^{i+}, m_{t}^{i-}\right)_{t \in \mathcal{T}}$ are essentially the same problem. Consequently, $\alpha_{i}^{(k) t}\left(p_{t}^{(k)}\right)$ can be optimized by using the following procedure in the $k$-th iteration:

$$
\begin{aligned}
x_{i}^{*}\left(p^{(k)}\right) & =\underset{x_{i} \in \mathcal{X}_{i}}{\operatorname{argmax}}\left(\phi_{i}\left(x_{i}\right)+\sum_{t \in \mathcal{T}} p_{t}^{(k)}\left(\gamma m_{i}^{t+}-m_{i}^{t-}\right)\right) \\
\alpha_{i}^{(k) t}\left(p_{t}^{(k)}\right) & =\beta_{i}^{(k) t} p_{t}^{(k)}+\left(m_{i}^{* t-}\left(p_{t}^{(k)}\right)-m_{i}^{* t+}\left(p_{t}^{(k)}\right)\right)
\end{aligned}
$$

Equation (31) corresponds to one of the optimal solutions of the sub-problem.

In the $k$-th iteration, each agent assumes that the price profile is $p^{(k)}:=\left(p_{t}^{(k)}\right)_{t \in \mathcal{T}}$. Each agent rationally maximizes its welfare, i.e., solves its sub-problem, obtains $x_{i}^{*}$ and determines $\alpha_{i}^{(k)}=\left(\alpha_{i}^{(k) t}\right)_{t \in \mathcal{T}}$. After all of the participating agents submit $\alpha_{i}^{(k)}$ to the auctioneer, the clearing price $p^{(k+1)}$ settled by market_clearing is determined based on Equation (29). The amount of electricity the $i$-th agent transacts through the regional market $\left(m_{i}^{t+}, m_{i}^{t-}\right)$ is determined based on $p^{(k+1)}$ :

$$
\left(m_{i}^{(k+1) t+}, m_{i}^{(k+1) t-}\right) \leftarrow\left(\mu_{i}^{t+}\left(p^{(k+1)}\right), \mu_{i}^{t-}\left(p^{(k+1)}\right)\right)
$$

As a result, the state vector of the $i$-th agent reactively becomes:

$$
\tilde{x}_{i}^{* t}=\left(l_{i}^{* t+}, l_{i}^{* t-}, b_{i}^{* t+}, b_{i}^{* t-}, m_{i}^{(k+1) t+}, m_{i}^{(k+1) t-}, g_{i}^{* t+}, g_{i}^{* t-}\right)
$$

However, this state vector is usually out of a feasible set, i.e., $\tilde{x}_{i}^{* t} \notin \mathcal{X}_{i}$. To solve the problem, a reconfiguration of $x_{i}^{t}$ is performed. The reconf $\mathcal{X}_{i}\left(\cdot \mid m_{i}^{t+}=m_{i}^{(k+1) t+}, m_{i}^{t-}=m_{i}^{(k+1) t-}\right)$ denotes a rational reconfiguration behavior of each agent that solves the sub-problem under the condition that, i.e., $\left(m_{i}^{t+}, m_{i}^{t-}\right)=\left(m_{i}^{(k+1) t+}, m_{i}^{(k+1) t-}\right)$, again.

\subsection{Convergence Proof of the Convergent Linear Function Submission-Based Double-Auction (CLFS-DA)}

In contrast with the fact that the LFS-DA mechanism does not have convergence proof, the CLFS-DA mechanism has convergence proof. The main theorem of this paper is as follows:

Theorem 1. By using the CLFS-DA mechanism, the price profile automatically converges to one of the optimal price profiles and social welfare is maximized if the sets of sellers and buyers for each time slot are fixed after a certain number of iterations $k_{0}$. 
Proof. In the LFS-DA, the price profile $p^{(k)}$ is updated in a similar way as RTP based on DD [21]. This property is obviously inherited in the CLFS-DA. The price profile of the CLFS-DA is updated as follows, when $k>k_{0}$ :

$$
\begin{aligned}
p_{t}^{(k+1)} & =p_{t}^{(k)}-\bar{\theta}_{k}^{t} \xi_{t}\left(p^{(k)}\right) \\
\xi_{t}\left(p^{(k)}\right) & :=\sum_{i \in \mathcal{N}} f_{i}^{t}\left(x_{i}^{* t}\left(p^{(k)}\right)\right) \\
& =\left(\sum_{i \in \mathcal{N}}\left(\gamma m_{i}^{* t+}\left(p^{(k)}\right)-m_{i}^{* t-}\left(p^{(k)}\right)\right)\right)_{t \in \mathcal{T}}
\end{aligned}
$$

where $\bar{\theta}_{k}^{t}=\left(\beta_{I_{t}\left(p_{t}^{(k+1)}\right)}^{(k) t}\right)^{-1}$, and $\beta_{I_{t}\left(p_{t}\right)}^{(k) t}=\gamma \beta_{I_{t}^{+}\left(p_{t}\right)}^{(k) t}+\beta_{I_{t}^{-}\left(p_{t}\right)}^{(k) t}$. The proof is provided in [21].

Based on the relationship between the LFS-DA and RTP based on dual decomposition with the sub-gradient method (RTP-DDSG) and Lemma 1, the following equation is satisfied:

$$
g_{\text {best }}^{(k)}-g^{*} \leq \frac{R^{2}+G^{2} \sum_{j=1}^{k}\left(\bar{\theta}_{j}^{t}\right)^{2}}{2 \sum_{j=1}^{k} \bar{\theta}_{j}^{t}}
$$

In this equation, $\gamma \in[0,1]$ yields:

$$
\begin{aligned}
\sum_{j=1}^{k}\left(\bar{\theta}_{j}^{t}\right)^{2} & =\sum_{j=1}^{k}\left(\left(\beta_{I_{t}\left(p_{t}^{(j+1)}\right)}^{(j) t}\right)^{-1}\right)^{2} \leq \sum_{j=1}^{k}\left(\left(\gamma \sum_{i=1}^{N} \beta_{i}^{(j) t}\right)^{-1}\right)^{2} \\
& =\sum_{j=1}^{k}\left(\left(\gamma \sum_{i=1}^{N} j v_{i}^{t}\right)^{-1}\right)^{2}=\left(\sum_{j=1}^{k} \frac{1}{j^{2}}\right)\left(\gamma \sum_{i=1}^{N} v_{i}^{t}\right)^{-2}, \text { and } \\
\sum_{j=1}^{k}\left(\bar{\theta}_{j}^{t}\right) & =\sum_{j=1}^{k}\left(\left(\beta_{\left.I_{t}\left(p_{t}^{(j+1)}\right)^{(j) t}\right)}\right)^{-1}\right) \sum_{j=1}^{k}\left(\left(\sum_{i=1}^{N} \beta_{i}^{(j) t}\right)^{-1}\right) \\
& =\sum_{j=1}^{k}\left(\left(\sum_{i=1}^{N} j v_{i}^{t}\right)^{-1}\right)=\left(\sum_{j=1}^{k} \frac{1}{j}\right)\left(\gamma \sum_{i=1}^{N} v_{i}^{t}\right)^{-1}
\end{aligned}
$$

These show that $\sum_{k=1}^{\infty}\left(\bar{\theta}_{k}^{t}\right)^{2}<\infty$ and $\sum_{k=1}^{\infty} \bar{\theta}_{k}^{t}=\infty$. Therefore, the price profile converges to one of the optimal values as $k \rightarrow \infty$.

Theorem 2. In the CLFS-DA mechanism, the determined optimal value does not depend on the initial selection of $\left(v_{i}^{t}\right)_{t \in \mathcal{T}, i \in \mathcal{N}}$.

Proof. Theorem 1 expresses that the iterative computation in the CLFS-DA mechanism converges to one of the optimal solutions of the dual problem because of its convexity. The optimal value of the dual problem is also an optimal value of the primal problem. The optimal value of the primal problem does not depend on $\left(v_{i}^{t}\right)_{t \in \mathcal{T}, i \in \mathcal{N}}$.

\subsection{Simple Convergent Linear Function Submission-Based Double-Auction (CLFS-DA)}

Theorem 2 also suggests that the solution of the primal problem determined by the CLFS-DA rarely depends on the agents' selection of $\left(v_{i}^{t}\right)_{t \in \mathcal{T}, i \in \mathcal{N}}$, practically. Based on Theorem 2, we can naturally introduce a simplified version of the CLFS-DA called the simple CFLS-DA (sCFLS-DA). In the sCLFS-DA, each agent does not need to submit $\left(v_{i}^{t}\right)_{t \in \mathcal{T}}$, and a predetermined default value $\bar{v}>0$ is used, i.e., $v_{i}^{t}=\bar{v} \forall t \in \mathcal{T}, \forall i \in \mathcal{N}$.

Theorem 3. By using the sCLFS-DA mechanism, the price profile automatically converges to one of the optimal prices, and social welfare is maximized. 
Proof. The sCLFS-DA is a type of CLFS-DA. Therefore, it is obviously followed by Theorem 1.

In sum, in the CLFS-DA, if the auctioneer simply determines a clearing price profile so as to balance supply and demand with an increasing $\beta_{i}^{t}$, i.e., $\beta_{i}^{t}=k v_{i}^{t}$ in the $k$-th iteration, it implicitly solves the dual problem iteratively by tuning the learning rate $\theta_{k}^{t}$ of the sub-gradient descent, so that the emergent searching algorithm converges to one of the optimal values in a bottom-up manner.

\section{Experiment}

This section demonstrates our numerical simulation of the CLFS-DA. Additionally, we compare CLFS-DA's results with those obtained by using the LFS-DA and the RTP-DDSG through a simulation experiment.

\subsection{Experimental Conditions}

The number of prosumers is $N=20$, and the number of time slots is $T=24$ in this experiment. The utility functions are $D_{i}^{t}(l):=\omega_{i}^{t} l-\frac{\kappa_{i}^{t}}{2} l^{2}$, if $0 \leq l \leq \frac{\omega_{i}^{t}}{\kappa_{i}^{t}}$, and otherwise $D_{i}^{t}(l):=\frac{\left(\omega_{i}^{t}\right)^{2}}{2 \kappa_{i}^{t}}$, where $\omega_{i}^{t}$ and $\kappa_{i}^{t}>0$ are the given constants $[4,40]$. We set $\kappa_{i}^{t}=10$ and $\omega_{i}^{t}=30$ for all $t \in \mathcal{T}, i \in \mathcal{N}$. We assumed all generators are PV cell systems; therefore, no variable costs were considered. The generation costs becomes $C_{i}^{t}\left(l_{i}^{t-}\right):=0 \quad\left(\forall l_{i}^{t-} \in\right.$ $\left.\left[0, l_{i}^{t-, \max }\right]\right)$. We used the PV cell generation profiles that were measured at 20 houses in Higashi Ohmi city during autumn of 2010 as the maximum value of the energy production profile $l_{i}^{t-, \max }$. Figure 2 shows $l_{i}^{t-, \max }$ for all prosumers. We set $s_{i}^{\text {init }}=0, s_{i}^{\max }=5, b_{i}^{t+, \max }=1$, $b_{i}^{t-, \max }=1, \eta_{i}=0.7, \gamma=0.8, m_{i}^{t+, \max }=5, m_{i}^{t-, \max }=5, p_{t}^{G-}=20$ and $p_{t}^{G+}=0$ for all $i \in \mathcal{N}, t \in \mathcal{T}$.

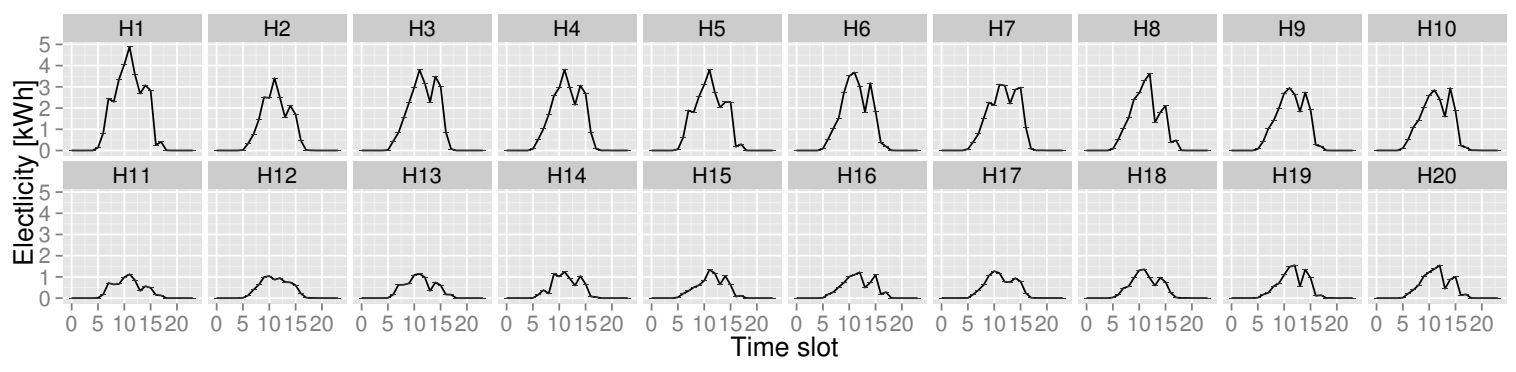

Figure 2. Electric energy generation profiles used in the experiment. The profiles are set to $l_{i}^{t-, \max }$. They were obtained from 20 prosumers' photovoltaic (PV) cells in Higashi Ohmi city, Japan [21].

In this experiment, the following comparative methods were prepared: the RTP-DDSG with fixed learning rate $\theta_{k}^{t}=0.01$, the RTP-DDSG with variable learning rate $\theta_{k}^{t}=0.1 \times \frac{1}{k}$ that satisfies the convergence condition, without trading, where each agent cannot trade among decentralized smart grid, the LFS-DA $\beta_{i}^{t}=5.0$, the CLFS-DA $v_{i}=0.05 \times i$ and the sCLFS-DA $\bar{v}=0.5$ for all $i \in \mathcal{N}$, $t \in \mathcal{T}$. The parameters related to the learning rate are selected so that the comparison becomes fair to some extent. In the RTP-DDSG, the supply and demand were usually not balanced because the network constraint was relaxed throughout the learning phase. In such a case, the central entity compensates the difference by selling the surplus electricity to or buying the deficit electricity from the outside grid. The cost was incurred equally for all agents afterward. We obtained the optimal one by solving the primal problem using a numerical solver.

\subsection{Results}

The overall performance of each mechanism is compared from the viewpoint of social welfare. The value of the social welfare is shown in Figure 3 as a function of each iteration step. The figure shows that the LFS-DA-based methods, i.e., the LFS-DA, CLFS-DA and sCLFS-DA, clearly 
outperform the RTP-DDSG. The LFS-DA-based methods immediately increase social welfare during the early stage of the iterative optimization process in a day-ahead market. The RTP-DDSG also gradually increases social welfare, but it contains undesirable oscillation. The RTP-DDSG decrees its social welfare with the cost of the compensation for the violation of the balance between demand and supply. The utility has to buy and sell electricity to bridge the gap between demand and supply, which affects overall social welfare negatively. Among LFS-DA-based methods, the CLFS-DA increases social welfare most smoothly and quickly. This finding means that the CLFS-DA is an effective mechanism for solving the primal problem for i-Rene.

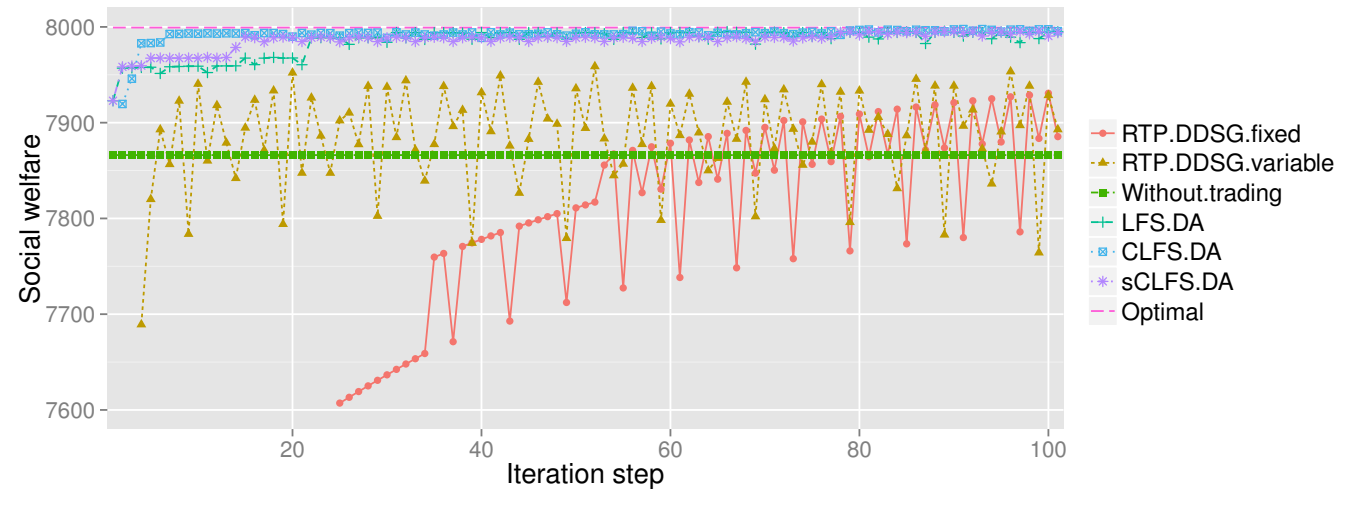

Figure 3. Social welfare after iterations with each method.

The most significant difference between the LFS-DA, a previously proposed method, and the CLFS-DA, the method proposed herein, is that the CLFS-DA has the property of convergence, as pointed out in Section 1. The convergence performance was investigated in Figure 4 . The convergence errors of the primal problem are plotted for each comparative method. This shows that the CLFS-DA and the sCLFS-DA make the convergence error approach zero gradually. A numerical demonstration shows that the CLFS-DA and the SCLFS-DA have better performance in terms of the convergence property than the LFS-DA, as our main theorem suggested.

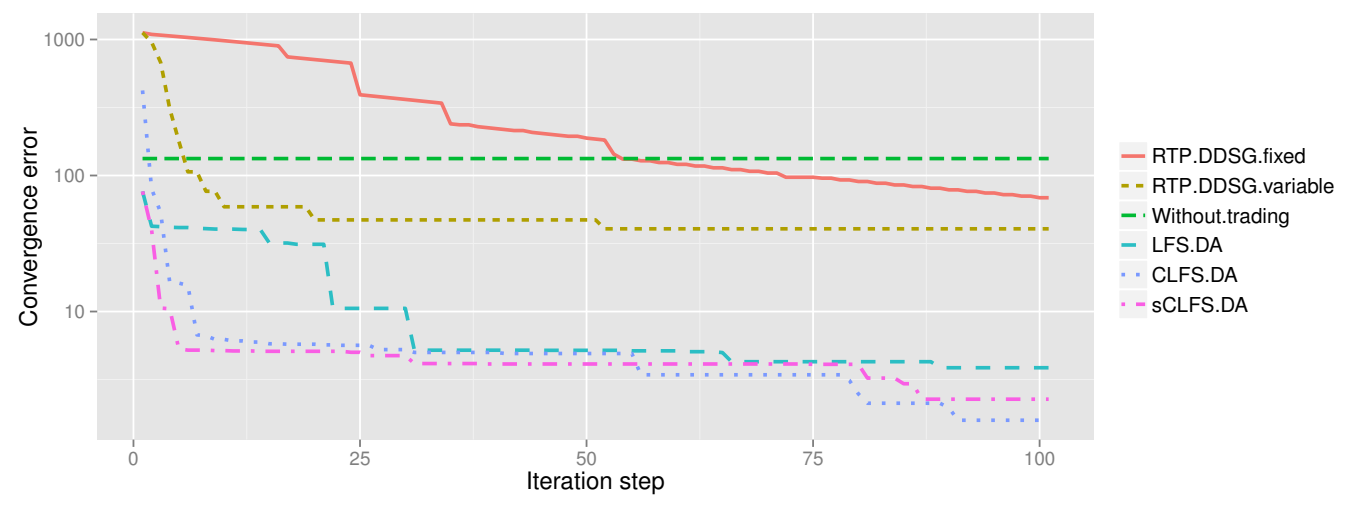

Figure 4. Convergence error after iterations with each method.

The lack of the property of convergence usually implies that the numerical solution converges slowly and continues to oscillate over time. Figure 5 shows the price profiles obtained by the LFS-DA and the CLFS-DA in each iteration between the first and 20th iterations. In Figure 5, although the highest prices in the LFS-DA and CLFS-DA differ, this does not cause any problems. Because no prices above $P_{t}^{G-}$ are used, the difference is essentially meaningless. This figure shows that the CLFS-DA quickly changed its price profile and almost converged to an appropriate price profile in only a few 
iterations in contrast to the LFS-DA, which gradually changed its price and still oscillated in the 20th iteration. To evaluate the oscillation that remained after a sufficient number of iterations, we calculated the average $\left|p^{(k+1)}-p^{(k)}\right|$ for the last 50 iterations. That of the LFS-DA was $6.51 \times 10^{-2}$, and that of the CLFS-DA was $6.92 \times 10^{-3}$. This finding clearly shows that the CLFS-DA has better performance in terms of convergence, as well as for the fast search of an adequate price profile. The above numerical results support our main theorem, i.e., Theorem 1.

LFS-DA
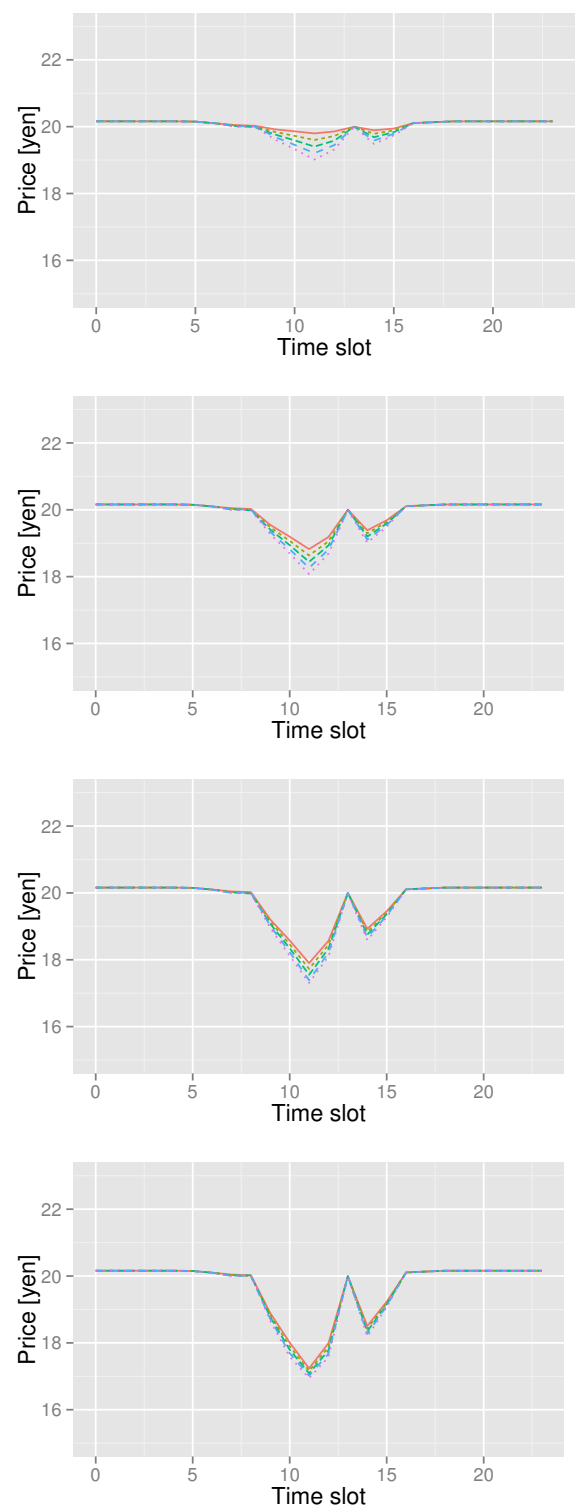

CLFS-DA
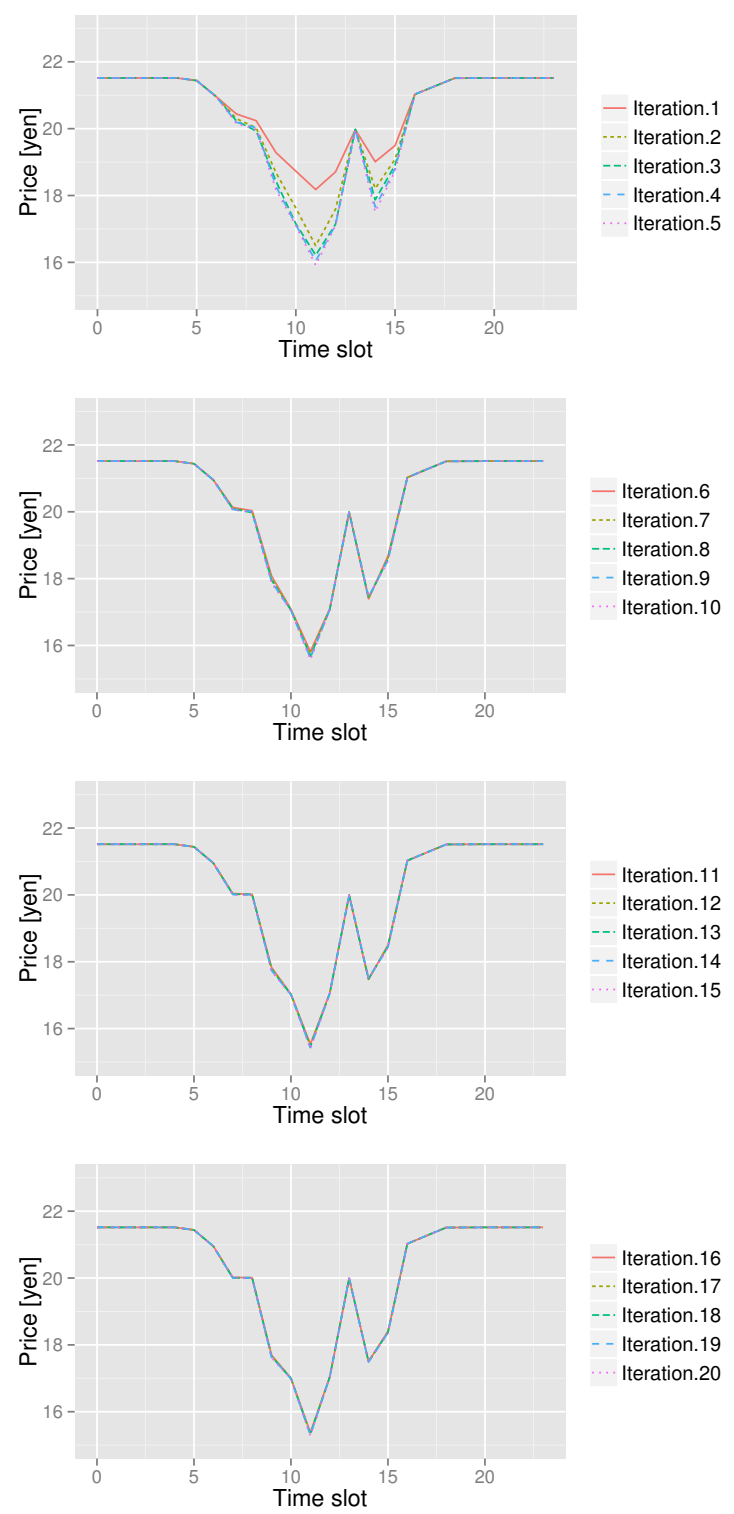

Figure 5. Price profiles obtained at each iteration when (left) the LFS-DA and (right) the CLFS-DA are used, respectively.

Next, Theorems 2 and 3 are investigated numerically. Theorem 2 suggests that the solutions obtained by the sCLFS-DA and the CLFS-DA become similar although $\left(v_{i}^{t}\right)_{t \in \mathcal{T}, i \in \mathcal{N}}$ are different, and therefore, numerical solutions in the early stage of the iterative process must be different. Figure 6 shows the individual welfare for each agent after the first iteration and that after the 100-th iteration. It shows that the differences among $\left(v_{i}^{t}\right)_{t \in \mathcal{T}, i \in \mathcal{N}}$ do not affect the final distribution of wealth. It suggests that each agent can determine $\left(v_{i}^{t}\right)_{t \in \mathcal{T}, i \in \mathcal{N}}$ selfishly, but it does not affect the agent's welfare if a 
sufficient number of iterations are performed. These numerical results support the effectiveness and reliability of the CLFS-DA and suggest the validity of the theorems provided in the previous section.

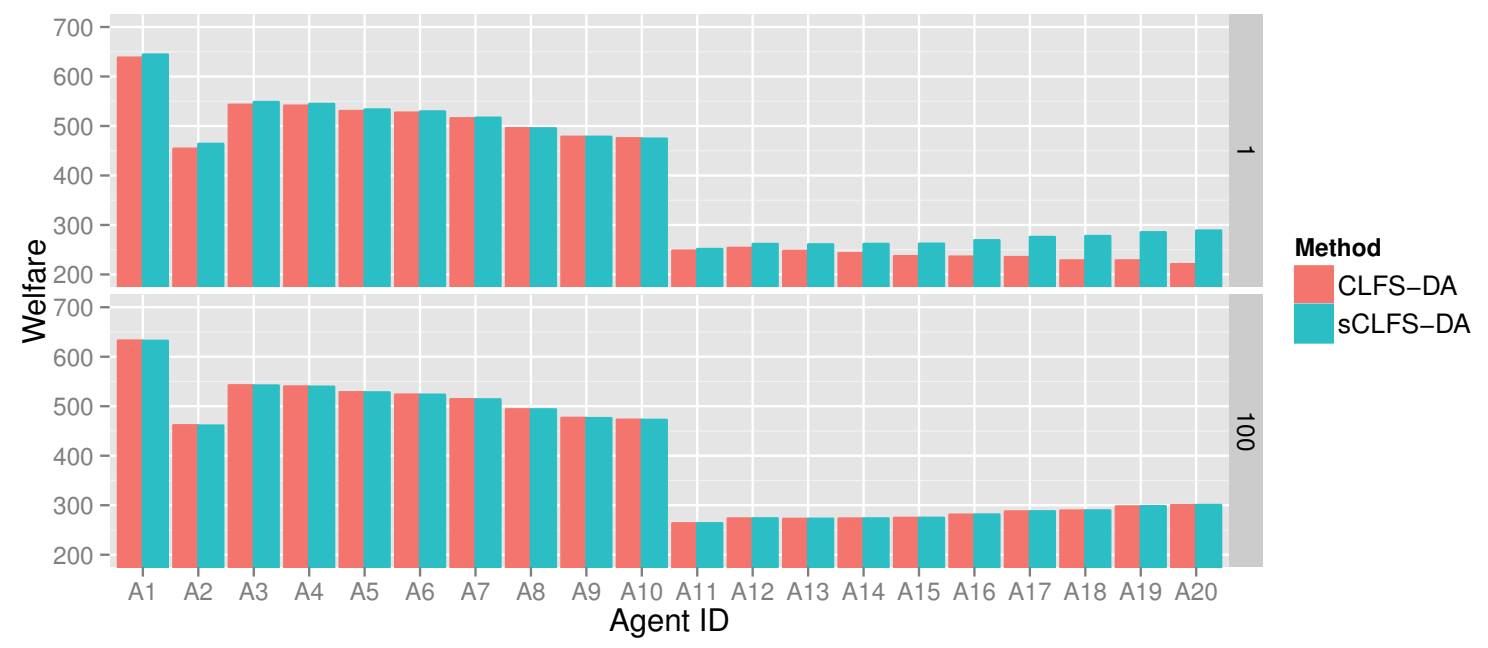

Figure 6. Individual welfare of each agent after the first iteration (top), and after the 100-th iteration (bottom).

\section{Conclusions}

In this paper, we described the CLFS-DA mechanism for a prosumers' decentralized smart grid called i-Rene. i-Rene consists of many prosumers equipped with a battery and a renewable energy-based generator, e.g., PVs. The CLFS-DA is an extension of the LFS-DA previously proposed double-auction mechanism in which each prosumer submits essentially linear demand and supply functions to an automated regional electricity market. As the main theorem in this paper, we proved that the CLFS-DA makes the price profile automatically converge to one of the optimal price profiles and maximizes the network's social welfare in contrast to the LFS-DA, which does not have such a convergence property. Unlike the LFS-DA, in the CLFS-DA mechanism, prosumers submit the initial slope of the bidding function $v_{i}^{t}$. Therefore, analyzing the effect of the selected $v_{i}^{t}$ on social welfare is important. Theorem 2 proved that the selection of $v_{i}^{t}$ does not affect the final social welfare obtained after convergence. Consequently, the sCLFS-DA was also proposed, and its convergence property was proven, as well (Theorem 3). Finally, on the basis of these theoretical results, we conducted an experiment, showing that the results were numerically supported.

The difference between CLFS-DA and LFS-DA might look simple and small at a glance, but it gives the significant and non-trivial difference to the new double auction mechanism, CLFS-DA. First, the iterative process of the CLFS-DA mechanism is guaranteed to converge to one of the optimal solutions of the original primal problem in contrast to the LFS-DA. Second, the numerical experiment showed that the CLFS-DA can find an appropriate price profile more promptly than the LFS-DA. In addition, the CLFS-DA inherits all of the advantageous characteristics of the LFS-DA as mentioned in Section 1. For example, the CLFS-DA mechanism also can achieve an exact balance between demand and supply for each time slot even through the learning phase in the same way as the LFS-DA. The performance of the proposed mechanism and its characteristics were demonstrated numerically through a simulation experiment. In this sense, the CLFS-DA is superior to the LFS-DA.

When we apply an iterative auction mechanism, e.g., RTP-DDSG, LFS-DA or CLFS-DA, to the real regional automated day-ahead market, the fast determination of the price profile and convergence without oscillation are very important. In the actual implementation, some costs, e.g., time and computation, are incurred for each iteration [17]. Therefore, the iterative process must be truncated on the basis of a predefined stopping criterion, as shown in Algorithm 1. Oscillation usually makes it difficult to satisfy the predefined stopping criterion. The faster determination of the 
appropriate price allows for the earlier truncation of the iterative process, which in turn results in a lower implicit system cost for the iteration process.

In the CLFS-DA, no prosumer can select $\beta_{i}^{t}$ directly in each step. He/she can determine only the initial slope of the bidding function $v_{i}^{t}$. If this restriction harms each agent's welfare, the mechanism might be unacceptable to prosumers in i-Rene. If the restriction harms the welfare of some prosumers, they will be against the introduction of the CLFS-DA and i-Rene. However, Theorem 2 suggests that the restriction does not affect the final welfare obtained by each agent. Theorem 2 is thus an important property when we attempt to implement the CLFS-DA in actual society.

Although the proposed CLFS-DA is a sophisticated mechanism, several actions are necessary to develop a reliable prosumers' regional smart grid managed automatically and efficiently in a decentralized manner. As many researchers have suggested, the FS-DA framework is very powerful for distributed optimization for the day-ahead market. By contrast, the real-time market is also important for managing a regional smart grid automatically in our actual environment because renewable energies and energy consumption have uncertainty and they cannot be predicted accurately on the previous day. Related works have proposed several market mechanisms for real-time electricity markets. Yo et al. [18] proposed the RTP-DDSG-based real-time market mechanism. Taniguchi and Yano [24] and Koket al. [36] applied LFS-DA-like double auction mechanisms to real-time markets heuristically, but they did not obtain theoretical results. Extending the CLFS-DA framework to a real-time market and to a stochastic environment for managing uncertainty in the regional prosumers' decentralized smart grid is one of our future directions. Testing our proposed mechanism in a real regional decentralized smart grid is also one of our future directions.

Acknowledgments: This research was partially supported by the Regional Innovation Strategy Support Program and Super Cluster Program (Satellite Shiga Area) funded by the Ministry of Education, Culture, Sports, Science and Technology, Japan.

Author Contributions: Tadahiro Taniguchi proved the main theorem and wrote the paper. Koki Kawasaki and Tomohiro Takata performed the experiments. Yoshiro Fukui derived the basic formulation.

\section{Nomenclature}

\begin{tabular}{ll}
\hline$\square$ Variables controlled by each agent (output) \\
\hline$l_{i}^{t+} \in\left[l_{i}^{t+, \min }, \infty\right)$ & Electric energy consumption profile \\
$l_{i}^{t-} \in\left[0, l_{i}^{t-, \max }\right)$ & Electric energy generation profile \\
$b_{i}^{t+} \in\left[0, b_{i}^{t+, \max }\right]$ & Battery charge profile \\
$b_{i}^{t-} \in\left[0, b_{i}^{t-}, \max \right]$ & Battery discharge profile \\
$m_{i}^{t+} \in\left[0, m_{i}^{t+, \max }\right]$ & Profile of electric energy sold to the local electricity market \\
$m_{i}^{t-} \in\left[0, m_{i}^{t-, \max }\right]$ & Profile of electric energy bought from the local electricity market \\
$g_{i}^{t+} \in[0, \infty)$ & Profile of electric energy sold to the outside grid \\
$g_{i}^{t-} \in\left[0, g_{i}^{-,}, \max \right]$ & Profile of electric energy bought from the outside grid \\
$x_{i}^{t}$ & Profile of state vector \\
$s_{i}^{t} \in\left[0, s_{i}^{\max }\right]$ & Profile of the state of charge (SOC) of the battery \\
$\alpha_{i}^{t}$ & Constant term of parameters of the bidding function \\
$v_{i}^{t}>0$ & Initial slope of the bidding function, i.e., $\beta^{(1)}=v_{i}^{t}$ \\
\hline$\square$ Variables determined by the market (output) \\
\hline$\beta_{i}^{t}>0$ & Primary coefficient term of parameters of the bidding function \\
$p_{t}$ & Price profile \\
\hline
\end{tabular}




\begin{tabular}{ll}
\hline$\square$ Fixed parameters and functions for each agent (input) \\
\hline$\eta_{i} \in[0,1]$ & Storage efficiency \\
$C_{i}^{t}$ & Cost function for generating electric energy \\
$D_{i}^{t}$ & Utility function for consuming electric energy \\
$\phi_{i}^{t}$ & Individual utility function \\
$W_{i}^{t}$ & Individual welfare function \\
\hline$\square$ Fixed parameters for the electricity network (input) \\
\hline$\gamma \in[0,1]$ & Electricity transmission efficiency \\
$p_{t}^{G+}$ & Price of electricity sold to the outside grid \\
$p_{t}^{G-}$ & Price of electricity bought from the outside grid \\
\hline
\end{tabular}

The superscript $t \in \mathcal{T}$ denotes the $t$-th time slot in a day. The subscript $i \in \mathcal{N}$ denotes the $i$-th agent in an electricity network.

\section{Appendix}

\section{A. Convergence Proof of the RTP-DDSG}

Convergence proof of the RTP-DDSG is provided as follows.

Lemma 1. If the RTP mechanism's learning rate is $\left(\theta_{k}^{t}\right)_{t \in \mathcal{T}}$, the convergence error of the master problem becomes:

$$
g_{\text {best }}^{(k)}-g^{*} \leq \frac{R^{2}+G^{2} \sum_{j=1}^{k}\left(\theta_{j}^{t}\right)^{2}}{2 \sum_{j=1}^{k} \theta_{j}^{t}}
$$

where $p^{*}$ is one of the optimal price profiles, $G$ is a constant satisfying $\left\|\xi_{t}(p)\right\| \leq G, R$ is a constant satisfying $\left\|p^{(1)}-p^{*}\right\| \leq R, g^{*}$ is the optimal value of Equation (22), and $g_{\text {best }}^{(k)}=\min \left\{g\left(p^{(1)}\right), \ldots, g\left(p^{(k)}\right)\right\}$.

This lemma can be naturally derived from the widely-known convergence theorem about the sub-gradient method. For example, [41] provides a clear explanation. $R$ and $G$ clearly exist in i-Rene.

Lemma 2. If the RTP mechanism's learning rate satisfies $\sum_{k=1}^{\infty}\left(\theta_{k}^{t}\right)^{2}<\infty$ and $\sum_{k=1}^{\infty} \theta_{k}^{t}=\infty$, variables updated by using the sub-gradient method converge, i.e., the price profile converges to one of the optimal price profiles, and social welfare is maximized.

Proof. The right-hand side of Equation (43) converges to zero as $k \rightarrow \infty$.

For example, $\theta_{k}^{t}=1 / k$ is one of the most famous candidates satisfying Lemma 2 . These lemmas are not the contribution of this paper, but are the prerequisites for our main theorem. Though this algorithm gives a feasible solution for the decentralized energy dispatch problem after iterative communications between a central entity and distributed agents, it cannot achieve an exact balance between supply and demand before converging to an optimal solution, i.e., before performing an infinite number of iterations.

Conflicts of Interest: The authors declare no conflict of interest.

\section{References}

1. Hommelberg, M.; Warmer, C.; Kamphuis, I.; Kok, J.; Schaeffer, G. Distributed control concepts using multi-agent technology and automatic markets: An indispensable feature of smart power grids. In Proceedings of the IEEE Power Engineering Society General Meeting, Tampa, FL, USA, 24-28 June 2007; pp. 1-7. 
2. Vytelingum, P.; Ramchurn, S.; Voice, T.; Rogers, A.; Jennings, N. Trading agents for the smart electricity grid. In Proceedings of the 9th International Conference on Autonomous Agents and Multiagent Systems, Toronto, Canada, 10-14 May 2010; Volume 1, pp. 897-904.

3. Alizadeh, M.; Li, X.; Wang, Z.; Scaglione, A.; Melton, R. Demand-side management in the smart grid: Information processing for the power switch. IEEE Signal Process. Mag. 2012, 29, 55-67.

4. Samadi, P.; Mohsenian-Rad, A.H.; Schober, R.; Wong, V.W.S.; Jatskevich, J. Optimal Real-Time Pricing Algorithm Based on Utility Maximization for Smart Grid. In Proceedings of the First IEEE International Conference on Smart Grid Communications, Gaithersburg, MD, USA, 4-6 October 2010; pp. 415-420.

5. Hammerstrom, D.; Brous, J.; Chassin, D.; Horst, G.; Kajfasz, R.; Michie, P.; Oliver, T.; Carlon, T.; Eustis, C.; Jarvegren, O.; et al. Pacific Northwest GridWise ${ }^{T M}$ Testbed Demonstration Projects Part II . Grid Friendly ${ }^{T M}$ Appliance Project; Pacific Northwest National Laboratory: Richland, WA, USA, 2007.

6. Graditi, G.; Ippolito, M.; Telaretti, E.; Zizzo, G. An Innovative Conversion Device to the Grid Interface of Combined RES-Based Generators and Electric Storage Systems. IEEE Trans. Ind. Electron. 2015, 62, 2540-2550.

7. Cosentino, V.; Favuzza, S.; Graditi, G.; Ippolito, M.G.; Massaro, F.; Riva Sanseverino, E.; Zizzo, G. Transition of a distribution system towards an active network. Part II: Economical analysis of selected scenario. In Proceedings of the 3rd International Conference on Clean Electrical Power: Renewable Energy Resources Impact, Ischia, Italy, 14-16 June 2011; pp. 15-20.

8. Dou, C.; Liu, B. Multi-Agent Based Hierarchical Hybrid Control for Smart Microgrid. IEEE Trans. Smart Grid 2013, 4, 771-778.

9. Molderink, A.; Bakker, V.; Bosman, M.G.C.; Hurink, J.L.; Smit, G.J.M. Management and control of domestic smart grid technology. IEEE Trans. Smart Grid 2010, 1, 109-119.

10. Roossien, B.; Hommelberg, M.; Warmer, C.; Kok, K.; Turkstra, J.W. Virtual power plant field experiment using 10 micro-CHP units at consumer premises. In Proceedings of the CIRED Seminar SmartGrids for Distribution, Frankfurt, France, 23-24 June 2008.

11. Di Silvestre, M.L.; Graditi, G.; Riva Sanseverino, E. A generalized framework for optimal sizing of distributed energy resources in micro-grids using an indicator-based swarm approach. IEEE Trans. Ind. Inform. 2014, 10, 152-162.

12. Di Somma, M.; Yan, B.; Bianco, N.; Graditi, G.; Luh, P.; Mongibello, L.; Naso, V. Operation optimization of a distributed energy system considering energy costs and exergy efficiency. Energy Convers. Manag. 2015, 103, 739-751.

13. Saad, W.; Han, Z.; Poor, H.V.; Basar, T. Game-theoretic methods for the smart grid: An overview of microgrid systems, demand-side management, and smart grid communications. IEEE Signal Process. Mag. 2012, 29, 86-105.

14. Hammerstrom, D.J.; Investigator, P.; Ambrosio, R.; Carlon, T.A.; Desteese, J.G.; Kajfasz, R.; Pratt, R.G. Pacific Northwest GridWise ${ }^{T M}$ Testbed Demonstration Projects Part I. Olympic Peninsula Project; Pacific Northwest National Laboratory: Richland, WA, USA, 2007.

15. Namerikawa, T.; Okubo, N.; Sato, R.; Okawa, Y. Real-Time Pricing Mechanism for Electricity Market With Built-In Incentive for Participation. IEEE Trans. Smart Grid 2015, 6, 2714-2724.

16. Vytelingum, P.; Voice, T.; Ramchurn, S.; Rogers, A.; Jennings, N. Agent-based micro-storage management for the smart grid. In Proceedings of the 9th International Conference on Autonomous Agents and Multiagent Systems, Toronto, Canada, 10-14 May 2010; Volume 1, pp. 39-46.

17. Weckx, S.; Member, S.; Hulst, R.D.; Claessens, B.; Driesen, J. Multi-agent charging of electric vehicles respecting distribution transformer loading and voltage limits. IEEE Trans. Smart Grid 2014, 5, 2857-2867.

18. Yo, M.; Ono, M.; Williams, B.C.; Adachi, S. Risk-limiting, Market-based Power Dispatch and Pricing. In Proceedings of the European Control Conference (ECC), Zurich, Switzerland, 17-19 July 2013; pp. 3038-3045.

19. Vogt, H.; Weiss, H.; Spiess, P.; Karduck, A. Market-based prosumer participation in the smart grid. In Proceedings of the 2010 4th IEEE International Conference on Digital Ecosystems and Technologies (DEST), Dubai, United Arab Emirates, 13-16 April 2010; pp. 592-597.

20. Ferruzzi, G.; Graditi, G.; Rossi, F.; Russo, A. Optimal Operation of a Residential Microgrid: The Role of Demand Side Management. Intell. Ind. Syst. 2015, 1, 61-82. 
21. Taniguchi, T.; Kawasaki, K.; Fukui, Y.; Takata, T.; Yano, S. Automated Linear Function Submission-based Double Auction as Bottom-up Real-Time Pricing in a Regional Prosumers' Electricity Network. Energies 2015, 8, 7381-7406.

22. Lampropoulos, I.; Vanalme, G.M.; Kling, W.L. A methodology for modeling the behavior of electricity prosumers within the smart grid. In Proceedings of the 2010 IEEE PES Innovative Smart Grid Technologies Conference Europe (ISGT Europe), Gothenburg, Sweden, 11-13 October 2010; pp. 1-8.

23. Tapscott, D.; Williams, A.D. Wikinomics: How Mass Collaboration Changes Everything; Penguin Publishing Group: London, UK, 2010.

24. Taniguchi, T.; Yano, S. Decentralized trading and demand side response in inter-intelligent renewable energy network. In Proceedings of the The 6th International Conference on Soft Computing and Intelligent Systems/The 13th International Symposium on Advanced Intelligent Systems (SCIS-ISIS), Kobe, Japan, 20-24 November 2012.

25. Dusonchet, L.; Ippolito, M.G.; Telaretti, E.; Zizzo, G. An optimal operating strategy for combined RES based Generators and Electric Storage Systems for load shifting applications. In Proceedings of the Fourth International Conference on Power Engineering, Energy and Electrical Drives (POWERENG), Istanbul, Turkey, 13-17 May 2013; pp. 552-557.

26. Graditi, G.; Luisa, M.; Silvestre, D.; Gallea, R.; Sanseverino, E.R. Heuristic-based shiftable loads optimal management in smart micro-grids. IEEE Trans. Ind. Inform. 2014, 11, 271-280.

27. Hansen, J.; Knudsen, J.; Kiani, A.; Annaswamy, A.; Stoustrup, J. A Dynamic Market Mechanism for Markets with Shiftable Demand Response. In Proceedings of the 19th IFAC World Congress, Cape Town, South Africa, 24-29 August, 2014; pp. 1873-1878.

28. Mohsenian-Rad, A.H.; Wong, V.W.; Jatskevich, J.; Schober, R. Optimal and Autonomous Incentive-Based Energy Consumption Scheduling Algorithm for Smart Grid. In Proceedings of the Innovative Smart Grid Technologies (ISGT), Gaithersburg, MD, USA, 19-21 Janurary 2010; pp. 1-6.

29. Okajima, Y.; Murao, T.; Hirata, K.; Uchida, K. Integration Mechanisms for LQ Energy Day-ahead Market Based on Demand Response. In Proceedings of the IEEE Conference on Control Applications (CCA), Juan Les Antibes, France, 8-10 October 2014.

30. Li, N.; Chen, L.; Low, S.H. Optimal demand response based on utility maximization in power networks. In Proceedings of the IEEE Power and Energy Society General Meeting, San Diego, CA, USA, 24-29 July 2011; pp. 1-8.

31. Gatsis, N.; Giannakis, G.B. Cooperative multi-residence demand response scheduling. In Proceedings of the 45th Annual Conference on Information Sciences and Systems, Baltimore, MD, USA, 23-25 March 2011; pp. 1-6.

32. Miyano, Y.; Namerikawa, T. Load Leveling Control by Real-Time Dynamical Pricing Based on Steepest Descent Method. In Proceedings of the SICE Annual Conference (SICE), Akita, Japan, 20-23 August 2012; pp. 131-136.

33. Disfani, V.R.; Fan, L.; Piyasinghe, L.; Miao, Z. Multi-agent control of community and utility using Lagrangian relaxation based dual decomposition. Electr. Power Syst. Res. 2014, 110, 45-54.

34. Kiani, A.; Annaswamy, A. Distributed hierarchical control for renewable energy integration in a Smart Grid. In Proceedings of the IEEE PES Innovative Smart Grid Technologies (ISGT), Washington, DC, USA, 16-18 January 2012, pp. 1-8.

35. Palomar, D. A tutorial on decomposition methods for network utility maximization. IEEE J. Sel. Areas Commun. 2006, 24, 1439-1451.

36. Kok, K.; Roossien, B.; Macdougall, P.; Pruissen, O.V. Dynamic Pricing by Scalable Energy Management Systems-Field Experiences and Simulation Results using PowerMatcher. In Proceedings of the IEEE Power and Energy Society General Meeting, San Diego, CA, USA, 22-26 July 2012; pp. 1-8.

37. Xu, Y.; Li, N.; Low, S. Demand Response With Capacity Constrained Supply Function Bidding. IEEE Trans. Power Syst. 2015, doi:10.1109/TPWRS.2015.2421932.

38. Papavasiliou, A.; Hindi, H.; Greene, D. Market-based control mechanisms for electric power demand response. In Proceedings of the 49th IEEE Conference on Decision and Control (CDC), Atlanta, GA, USA, 15-17 December 2010; pp. 1891-1898.

39. Bertsekas, D.P. Nonlinear Programming; Athena Scientific: Nashua, NH, USA, 1999. 
40. Fahrioglu, M.; Alvarado, F.L. Using utility information to calibrate customer demand management behavior models. IEEE Trans. Power Syst. 2001, 16, 317-322.

41. Boyd, S.; Xiao, L.; Mutapcic, A. Subgradient methods; Stanford University, Stanford, CA, USA, 2008.

(C) 2015 by the authors; licensee MDPI, Basel, Switzerland. This article is an open access article distributed under the terms and conditions of the Creative Commons by Attribution (CC-BY) license (http://creativecommons.org/licenses/by/4.0/). 